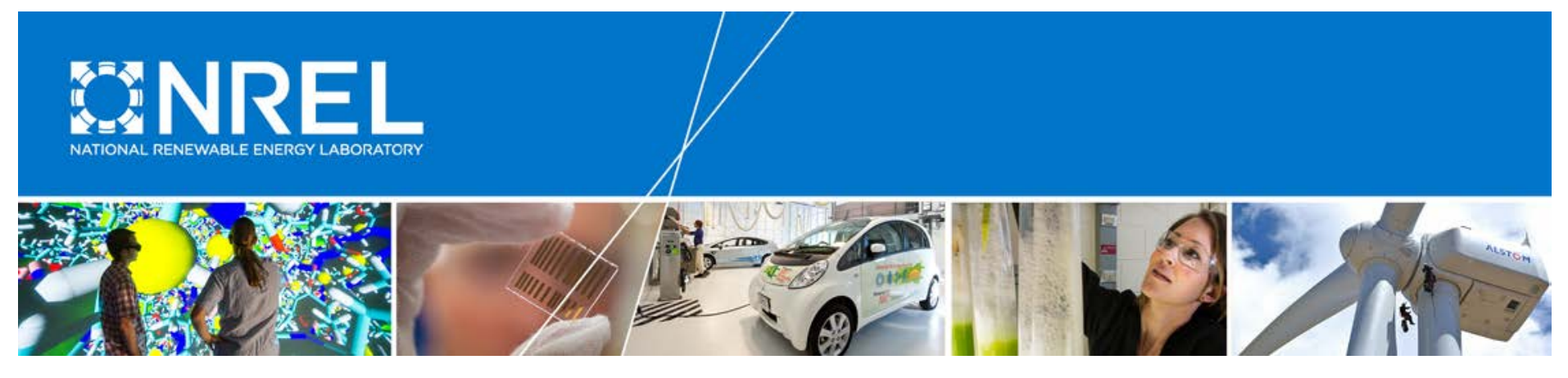

\title{
Optimal Power Flow for Distribution Systems under Uncertain Forecasts
}

\section{Preprint}

\author{
Emiliano Dall'Anese and Kyri Baker \\ National Renewable Energy Laboratory \\ Tyler Summers \\ University of Texas at Dallas
}

To be presented at the 55th IEEE Conference on Decision and Control

Las Vegas, Nevada

December 12-14, 2016

(C) 2016 IEEE. Personal use of this material is permitted. Permission from IEEE must be obtained for all other uses, in any current or future media, including reprinting/republishing this material for advertising or promotional purposes, creating new collective works, for resale or redistribution to servers or lists, or reuse of any copyrighted component of this work in other works.

NREL is a national laboratory of the U.S. Department of Energy Office of Energy Efficiency \& Renewable Energy Operated by the Alliance for Sustainable Energy, LLC

This report is available at no cost from the National Renewable Energy Laboratory (NREL) at www.nrel.gov/publications.

\section{Conference Paper}

NREL/CP-5D00-66856

December 2016 


\section{NOTICE}

The submitted manuscript has been offered by an employee of the Alliance for Sustainable Energy, LLC (Alliance), a contractor of the US Government under Contract No. DE-AC36-08GO28308. Accordingly, the US Government and Alliance retain a nonexclusive royalty-free license to publish or reproduce the published form of this contribution, or allow others to do so, for US Government purposes.

This report was prepared as an account of work sponsored by an agency of the United States government. Neither the United States government nor any agency thereof, nor any of their employees, makes any warranty, express or implied, or assumes any legal liability or responsibility for the accuracy, completeness, or usefulness of any information, apparatus, product, or process disclosed, or represents that its use would not infringe privately owned rights. Reference herein to any specific commercial product, process, or service by trade name, trademark, manufacturer, or otherwise does not necessarily constitute or imply its endorsement, recommendation, or favoring by the United States government or any agency thereof. The views and opinions of authors expressed herein do not necessarily state or reflect those of the United States government or any agency thereof.

This report is available at no cost from the National Renewable Energy Laboratory (NREL) at www.nrel.gov/publications.

Available electronically at SciTech Connect http:/www.osti.gov/scitech

Available for a processing fee to U.S. Department of Energy and its contractors, in paper, from:

U.S. Department of Energy

Office of Scientific and Technical Information

P.O. Box 62

Oak Ridge, TN 37831-0062

OSTI http://www.osti.gov

Phone: 865.576.8401

Fax: 865.576.5728

Email: reports@osti.gov

Available for sale to the public, in paper, from:

U.S. Department of Commerce

National Technical Information Service

5301 Shawnee Road

Alexandria, VA 22312

NTIS http://www.ntis.gov

Phone: 800.553 .6847 or 703.605 .6000

Fax: 703.605.6900

Email: orders@ntis.gov 


\title{
Optimal Power Flow for Distribution Systems Under Uncertain Forecasts
}

\author{
Emiliano Dall'Anese, Kyri Baker, and Tyler Summers
}

\begin{abstract}
This paper focuses on distribution systems featuring renewable energy sources and energy storage devices, and develops an optimal power flow (OPF) approach to optimize the system operation in spite of forecasting errors. The proposed method builds on a chance-constrained multi-period AC OPF formulation, where probabilistic constraints are utilized to enforce voltage regulation with a prescribed probability. To enable a computationally affordable solution approach, a convex reformulation of the OPF task is obtained by resorting to i) suitable linear approximations of the power flow equations, and ii) convex approximations of the chance constraints. Particularly, the approximate chance constraints provide conservative bounds that hold for arbitrary distributions of the forecasting errors. An adaptive optimization strategy is then obtained by embedding the proposed OPF task into a model predictive control framework.
\end{abstract}

\section{INTRODUCTION}

The increased deployment of renewable energy sources (RESs) with business-as-usual practices have already precipitated a unique set of power-quality and reliability concerns at the distribution-system level. Reverse power flows increase the likelihood of voltages violating prescribed security limits, while rapid variations in RES generation perpetuate transients that ultimately lead to increased cycling and wearout of legacy voltage regulation equipment. Leveraging the increased flexibility offered by RES-inverters, local control strategies as well as network optimization approaches [1]-[5] are currently under development to alleviate these emerging concerns. Particularly, optimization tasks can be carried out by advance distribution management systems (ADMSs), which leverage the underlying communication infrastructure to broadcast power commands. RES-inverters can be controlled alongside energy storage units to minimize curtailment of renewable-based generation, shape the net load profile in response to market signals, and provide ancillary services to the main grid [6].

The paper focuses on network optimization strategies to systematically dispatch the setpoints of RESs and energy storage systems based on forecasts of ambient conditions and loads. The proposed approach builds on a model predictive control (MPC) technique, where optimal setpoints for RESinverters and energy storage units are obtained at each point in time by solving a multi-period AC optimal power flow $(\mathrm{OPF})$ problem. Examples of prior efforts that are aligned

E. Dall'Anese and K. Baker are with the National Renewable Energy Laboratory (NREL). T. Summers is with the Department of Mechanical Engineering, University of Texas at Dallas. E-mails: \{emiliano.dallanese, kyri.baker\}@nrel.gov, ths150130@utdallas.edu

The work of E. Dall'Anese and K. Baker was supported in part by the Laboratory Directed Research and Development Program at NREL. with the objectives of this paper include e.g., [1], where two-stage stochastic programming is utilized to solve an economic dispatch (based on a DC model) problem for microgrids, and [5], [7], where MPC strategies are utilized to dispatch energy storage commands. However, the approaches in [5], [7] do not model forecasting errors and utilize a DC model. A distributed AC OPF problem with MPC is solved in [8]; however, the forecasts are assumed perfect. Presuming a linear RES-control policy during the realtime operation, a robust multi-period DC OPF problem is formulated in [9], while chance-constrained problem setups are considered in [4], [10], [11]. The approach of [4] enables a deterministic reformulation of the chance constraints when forecast errors are Gaussian distributed, while [10], [11] leverage conservative convex approximations of the chance constraints. Finally, general control policies are considered in [12], and deterministic reformulations of the probabilistic constraints are derived for Gaussian-distributed forecast errors. Overall, [4], [10]-[12] offer means to deal with chance constraints in a computationally tractable way, but their applicability is limited to DC models. AC power flow models are considered in [13] where, however, forecasting errors are neglected, and in [14], where RES-inverter commands are dispatched based on conditional value-at-risk arguments.

The optimization method proposed in this paper utilizes a multi-period AC OPF formulation, where probabilistic constraints are utilized to enforce voltage regulation with a prescribed probability. Nonlinearity of AC power flow equations and probabilistic constraints render the formulated AC OPF task computationally intractable [15]. However, to enable a computationally affordable solution approach, an approximate reformulation of the AC OPF task is obtained by utilizing: i) the linear approximation of the $\mathrm{AC}$ power flow equations recently proposed in [16]; and, ii) pertinent convex approximations of the chance constraints [15]. The linear power-voltage relationship proposed in [16] yields markedly lower approximation errors compared to the DC model, and captures variations in voltage magnitudes due to changes in real and reactive power injections. On the other hand, the approximate chance constraints provide conservative bounds that hold for arbitrary distributions of the forecasting errors, and render the overall problem deterministic and convex.

\section{Preliminaries And System Model}

Consider a distribution feeder comprising $N+1$ nodes collected in the set $\mathcal{N} \cup\{0\}, \mathcal{N}:=\{1, \ldots, N\}$, and lines 
represented by the set of edges $\mathcal{E}:=\{(m, n)\} \subset \mathcal{N} \times \mathcal{N}$. Node 0 denotes the secondary of the distribution transformer. Let $V_{n}^{t} \in \mathbb{C}$ and $I_{n}^{t} \in \mathbb{C}$ denote the phasors for the lineto-ground voltage and the current injected at node $n \in \mathcal{N}$ at time $t$, respectively, and define $\mathbf{i}^{t}:=\left[I_{1}^{t}, \ldots, I_{N}^{t}\right]^{\top} \in$ $\mathbb{C}^{N}$ and $\mathbf{v}^{t}:=\left[V_{1}^{t}, \ldots, V_{N}^{t}\right]^{\top} \in \mathbb{C}^{N}$. Using Ohm's and Kirchhoff's circuit laws, the following linear relationship can be established

$$
\left[\begin{array}{c}
I_{0}^{t} \\
\mathbf{i}^{t}
\end{array}\right]=\underbrace{\left[\begin{array}{cc}
y_{0 j} & \overline{\mathbf{y}}^{\top} \\
\overline{\mathbf{y}}_{0} & \mathbf{Y}
\end{array}\right]}_{:=\mathbf{Y}_{\text {net }}}\left[\begin{array}{l}
V_{0}^{t} \\
\mathbf{v}^{t}
\end{array}\right]
$$

where the system admittance matrix $\mathbf{Y}_{\text {net }} \in \mathbb{C}^{N+1 \times N+1}$ is formed based on the system topology and the $\pi$-equivalent circuit of the distribution lines; see [17, Chapter 6] for additional details on distribution line modeling. Particularly, with $y_{m n}$ and $y_{m n}^{\mathrm{sh}}$ denoting the series and shunt admittances of line $(m, n)$, the entries of $\mathbf{Y}$ are formed as:

$$
\left[\mathbf{Y}_{\text {net }}\right]_{m, n}:= \begin{cases}\sum_{j \in \mathcal{N}_{m}} y_{m j}^{\mathrm{sh}}+y_{m j}, & \text { if } m=n \\ -y_{m n}, & \text { if }(m, n) \in \mathcal{E} \\ 0, & \text { otherwise }\end{cases}
$$

where $\mathcal{N}_{m}:=\{j \in \mathcal{N}:(m, j) \in \mathcal{E}\}$ is the set of nodes connected to the $m$-th one through a distribution line. For simplicity of exposition, assume that the shunt admittances $y_{m n}^{\text {sh }}$ are negligible [17].

A constant- $P Q$ model is adopted for the loads, with $P_{\ell, n}^{t}$ and $Q_{\ell, n}^{t}$ denoting the real and reactive demands at node $n \in \mathcal{N}$ at time $t$, respectively. Let $\mathbf{p}_{\ell}^{t}:=\left[P_{\ell, 1}^{t}, \ldots, P_{\ell, N}^{t}\right]^{\top}$ and $\mathbf{q}_{\ell}^{t}:=\left[Q_{\ell, 1}^{t}, \ldots, Q_{\ell, N}^{t}\right]^{\top}$ collect the loads; if no load is present at node $n \in \mathcal{N}$, then $P_{\ell, n}^{t}=Q_{\ell, n}^{t}=0, \forall t$.

RES model. For given ambient conditions, let $P_{\mathrm{av}, n}^{t}$ denote the maximum renewable-based real power generation at node $n \in \mathcal{N}$ at time $t-$ hereafter referred to as the available real power. Real power curtailment has been proposed as a means for overvoltage prevention [2], [3]; this strategy is particularly effective in systems where distribution lines have a high resistance-to-reactance ratio, where voltage magnitudes are mainly sensitive to changes in the net real power injections [14]. To account for the ability of the RES inverters to adjust the output real power, let $\alpha_{n}^{t} \in[0,1]$ denote the fraction of available real power curtailed by RES-inverters $n$ at time $t$; thus, the real power curtailed from $\operatorname{RES} n$ at times $t$ amounts to $\alpha_{n}^{t} P_{\mathrm{av}, n}^{t}$. For future developments, it is convenient to define the vectors $\boldsymbol{\alpha}^{t}:=\left[\alpha_{1}^{t}, \ldots, \alpha_{N}^{t}\right]^{\top}$ and $\mathbf{p}_{\mathrm{av}}^{t}:=\left[P_{\mathrm{av}, 1}^{t}, \ldots, P_{\mathrm{av}, N}^{t}\right]^{\top}$, with the convention that

\footnotetext{
${ }^{1}$ Upper-case (lower-case) boldface letters will be used for matrices (column vectors); $(\cdot)^{\top}$ for transposition; $(\cdot)^{*}$ complex-conjugate; and, $(\cdot)^{\mathrm{H}}$ complex-conjugate transposition; $\Re\{\cdot\}$ and $\Im\{\cdot\}$ denote the real and imaginary parts of a complex number, respectively; $\mathrm{j}:=\sqrt{-1}$ the imaginary unit; $|\cdot|$ denotes the absolute value of a number or the cardinality of a set. For $x \in \mathbb{R}$, function $[x]_{+}$is defined as $[x]_{+}:=\max \{0, x\}$. Further, $\mathbb{I}_{A}(x)$ denotes the indicator function over the set $A \subset \mathbb{R}$; that is $\mathbb{I}_{A}(x)=1$ if $x \in A$ and $\mathbb{I}_{A}(x)=0$ otherwise. For a given $N \times 1$ vector $\mathbf{x} \in \mathbb{R}^{N}$, $\|\mathbf{x}\|_{2}:=\sqrt{\mathbf{x}^{\mathrm{H}} \mathbf{x}} ;\|\mathbf{x}\|_{1}:=\sum_{i}\left|[\mathbf{x}]_{i}\right| ;$ and, $\operatorname{diag}(\mathbf{x})$ returns a $N \times N$ matrix with the elements of $\mathbf{x}$ in its diagonal. Finally, $\mathbf{I}_{N}$ denotes the $N \times N$ identity matrix; and, $\mathbf{0}_{M \times N}, \mathbf{1}_{M \times N}$ the $M \times N$ matrices with all zeroes and ones, respectively.
}

$\alpha_{n}^{t}=0$ and $P_{\mathrm{av}, n}^{t}=0$ if no RES is located at node $n$. Although this paper focuses on real power curtailment, reactive power compensation [2] can be easily incorporated in the framework presented subsequently.

Energy storage model. Let $B_{n}^{t}$ represent the state of charge (SoC) of an energy storage system located at node $n \in \mathcal{N}$, with the corresponding dynamical equation given by:

$$
B_{n}^{t+1}=B_{n}^{t}+P_{B, n}^{t} \Delta^{t}
$$

where $\Delta^{t}$ is the duration of slot $(t, t+1]$ and $P_{B, n}^{t}$ represents the power delivered to (drawn from) the storage device; particularly, $P_{B, n}^{t}$ commands either the charging $\left(P_{B, n}^{t}>0\right)$ or the discharging $\left(P_{B, n}^{t}<0\right)$ of the battery during slot $(t, t+1]$. The operational limits of the storage device are as follows:

$$
\begin{aligned}
& P_{B, n}^{\min } \leq P_{B, n}^{t} \leq P_{B, n}^{\max } \\
& B_{n}^{\min } \leq B_{n}^{t+1} \leq B_{n}^{\max }
\end{aligned}
$$

where $B_{n}^{\min }, B_{n}^{\max }$ are predetermined minimum and maximum SoC levels; $P_{B, n}^{\min }$ and $P_{B, n}^{\max }$ are minimum and maximum capacity limits. Constraints in (4b) indicate that a fraction of the stored energy (available storage) is available for discharge (charge). For future developments, define the vector $\mathbf{p}_{B}^{t}:=\left[P_{B, 1}^{t}, \ldots, P_{B, N}^{t}\right]^{\top}$.

Forecasting error model. The optimization problem that will be formulated in Section III considers a planning horizon $\mathcal{T}_{t}:=\{t, t+1, \ldots, t+T\}$ of $T$ discrete time steps. Given prevailing ambient and load conditions, $\mathbf{p}_{\mathrm{av}}^{t}, \mathbf{p}_{\ell}^{t}$, and $\mathbf{q}_{\ell}^{t}$ are assumed to be known [2], [5], [13]. On the other hand, to capture forecasting errors over the (future) time slots $\tau \in \mathcal{T}_{t}^{\prime}:=\{t+1, \ldots, t+T\}, \mathbf{p}_{\mathrm{av}}^{\tau}, \mathbf{p}_{\ell}^{\tau}$, and $\mathbf{q}_{\ell}^{\tau}$ are modeled as random variables [11]. Particularly, the available RES powers at time $\tau$ are modeled as $\mathbf{p}_{\mathrm{av}}^{\tau}=\overline{\mathbf{p}}_{\mathrm{av}}^{\tau}+\boldsymbol{\delta}_{\mathrm{av}}^{\tau}$, where $\overline{\mathbf{p}}_{\mathrm{av}}^{\tau} \in \mathbb{R}^{N}$ collects the forecasted values and $\boldsymbol{\delta}_{\mathrm{av}}^{\tau} \in \mathcal{R}_{\mathrm{av}}^{\tau} \subseteq \mathbb{R}^{N}$ is a random vector whose distribution captures spatial dependencies among forecasting errors. Similarly, the real and reactive loads at time $\tau \in \mathcal{T}_{t}^{\prime}$ are expressed as $\mathbf{p}_{\ell}^{\tau}=\overline{\mathbf{p}}_{\ell}^{\tau}+\mathbf{G}_{p}^{\tau} \boldsymbol{\delta}_{\ell}^{\tau}$ and $\mathbf{q}_{\ell}^{\tau}=\overline{\mathbf{q}}_{\ell}^{\tau}+\mathbf{G}_{q}^{\tau} \boldsymbol{\delta}_{\ell}^{\tau}$, respectively, where $\overline{\mathbf{p}}_{\ell}^{\tau}$ and $\overline{\mathbf{q}}_{\ell}^{\tau}$ are the forecasted loads; $\mathbf{G}_{p}^{\tau}, \mathbf{G}_{q}^{\tau} \in \mathbb{R}^{N \times N_{\ell}}$ are model-dependent matrices, with $N_{\ell}$ the dimension of the random vector; and, $\boldsymbol{\delta}_{\ell}^{\tau} \in \mathcal{R}_{\ell}^{\tau} \subseteq \mathbb{R}^{N_{\ell}}$ is a random vector whose distribution captures spatial dependencies as well as correlations among real and reactive loads. We assume that the a certain amount of information about the probability distributions of the forecasting errors $\boldsymbol{\delta}_{\mathrm{av}}^{\tau}$ and $\boldsymbol{\delta}_{\ell}^{\tau}$ is available [4], [10], [11], [14]. In particular, this information can come in the form of either knowledge of the probability density functions, knowledge of means and covariance matrices, or a model of $\boldsymbol{\delta}_{\mathrm{av}}^{\tau}$ and $\boldsymbol{\delta}_{\ell}^{\tau}$ from which one can draw samples.

\section{A. Leveraging approximate power-flow models}

Overall, power balance at node $n$ and time $t$ amounts to

$$
V_{n}^{t} \sum_{m \in \mathcal{N}_{n}} y_{n m}^{*}\left(V_{n}^{t}-V_{m}^{t}\right)^{*}=S_{n}^{t}
$$

where $S_{n}^{t}=\left(1-\alpha_{n}^{t}\right) P_{\mathrm{av}, n}^{t}-P_{\ell, n}^{t}-P_{B, n}^{t}-\mathrm{j} Q_{\ell, n}^{t}$. This equation typically appears in the form of a constraint in 
standard formulations of the OPF task, and renders the underlying optimization problem nonconvex [18]. Another source of nonconvexity in various OPF renditions is represented by the voltage-related constraint $V_{\min } \leq\left|V_{n}^{t}\right|$, where $V_{\text {min }}$ represents a pre-determined lower limit for the voltage magnitude (e.g., ANSI C84.1 limits). Semidefinite relaxation techniques have been employed to bypass the nonconvexity of voltage-regulation and power-balance constraints, and yet achieve globally optimal solutions of the nonconvex OPF under certain conditions (see e.g., [2], [18] and references therein). However, to develop a multi-period OPF formulation that is computationally affordable, linear surrogates of (5) and voltage-regulation constraints will be sought next. Approximate power-flow relations will also facilitate the application of convex approximation of chance constraints to the problem that will be formulated in Section III.

Without loss of generality, node 0 is taken to be the slack node, and the voltage $V_{0}^{t}=\rho_{0}^{t} e^{\mathrm{j} \theta_{0}^{j}}$ is assumed known. Let $\mathbf{s}^{t}:=\left(\mathbf{I}_{N}-\operatorname{diag}\left\{\boldsymbol{\alpha}^{t}\right\}\right) \mathbf{p}_{\mathrm{av}}^{t}-\mathbf{p}_{\ell}^{t}-\mathbf{p}_{B}^{t}-\mathbf{j} \mathbf{q}_{\ell}^{t}$ denote the vector of complex power injections. The voltages $\mathbf{v}^{t}$ satisfying the nonlinear power-balance equations (5) are expressed as $\mathbf{v}^{t}=\overline{\mathbf{v}}^{t}+\mathbf{e}^{t}$, where the entries of $\mathbf{e}^{t}$ capture deviations around the linearization points $\overline{\mathbf{v}}^{t}$. Collect in the vector $\overline{\boldsymbol{\rho}}^{t} \in \mathbb{R}_{+}^{N}$ the magnitudes of voltages $\overline{\mathbf{v}}^{t}$, and let $\overline{\boldsymbol{\gamma}}^{t} \in \mathbb{R}^{N}$ and $\overline{\boldsymbol{\mu}}^{t} \in \mathbb{R}^{N}$ collect elements $\left\{\cos \left(\bar{\theta}_{n}^{t}\right)\right\}$ and $\left\{\sin \left(\bar{\theta}_{n}^{t}\right)\right\}$, respectively, where $\bar{\theta}_{i}^{t}$ is the angle of the nominal voltage $\bar{V}_{i}^{t}$. Expanding on (5), and discarding second-order terms such as $\operatorname{diag}\left(\mathbf{e}^{t}\right) \mathbf{Y}^{*} \mathbf{e}^{t *}$, it turns out that (5) can be approximated as $\boldsymbol{\Gamma}^{t} \mathbf{e}^{t}+\boldsymbol{\Phi}^{t}\left(\mathbf{e}^{t}\right)^{*}=\mathbf{s}^{t}+\boldsymbol{v}^{t}$, where $\boldsymbol{\Gamma}^{t}:=\operatorname{diag}\left(\mathbf{Y}^{*} \overline{\mathbf{v}}^{t *}+\overline{\mathbf{y}}^{*} V_{0}^{t *}\right), \boldsymbol{\Phi}^{t}:=\operatorname{diag}\left(\overline{\mathbf{v}}^{t}\right) \mathbf{Y}^{*}$, and $\boldsymbol{v}^{t}:=-\operatorname{diag}\left(\overline{\mathbf{v}}^{t}\right)\left(\mathbf{Y}^{*} \overline{\mathbf{v}}^{t *}+\overline{\mathbf{y}}^{*} V_{0}^{t *}\right)$. Next, consider then the following choice of the nominal voltage $\overline{\mathbf{v}}^{t}$ :

$$
\overline{\mathbf{v}}^{t}=-\mathbf{Y}^{-1} \overline{\mathbf{y}} V_{0}^{t} .
$$

Using (6), it follows that $\boldsymbol{\Gamma}=\mathbf{0}_{N \times N}$ and $\boldsymbol{v}=\mathbf{0}_{N}$, and therefore one obtains the linearized power-flow expression

$$
\operatorname{diag}\left(\overline{\mathbf{v}}^{t *}\right) \mathbf{Y} \mathbf{e}^{t}=\mathbf{s}^{t *}
$$

Notice that matrix $\mathbf{Y}$ is diagonally dominant and irreducible [16]. Particularly, it is diagonally dominant by construction since $\left|y_{i i}\right| \geq \sum_{n \neq i}\left|y_{i n}\right|$ for all $n \in \mathcal{N}$; it is also irreducibly diagonally dominant if $\left|y_{0 i}\right|>0$ for any $i$. Then, a solution to (7) can be expressed as $\mathbf{e}^{t}=$ $\mathbf{Y}^{-1}\left(\operatorname{diag}\left(\overline{\mathbf{v}}^{t *}\right)\right)^{-1} \mathbf{s}^{t *}$. Thus, expanding on this relation, and assuming that angles $\bar{\theta}_{n}^{t}$ are small, an approximate voltagepower relationship

$$
\boldsymbol{\rho}^{t} \approx \mathbf{g}_{\rho}\left(\mathbf{p}^{t}, \mathbf{q}^{t}\right):=\mathbf{R}^{t} \mathbf{p}^{t}+\mathbf{B}^{t} \mathbf{q}^{t}+\mathbf{a}
$$

can be obtained by defining the matrices:

$$
\begin{aligned}
& \mathbf{R}^{t}=\mathbf{Z}_{R} \operatorname{diag}\left(\overline{\boldsymbol{\gamma}}^{t}\right) \\
& \quad \times\left(\operatorname{diag}\left(\overline{\boldsymbol{\rho}}^{t}\right)\right)^{-1}-\mathbf{Z}_{I} \operatorname{diag}\left(\overline{\boldsymbol{\mu}}^{t}\right)\left(\operatorname{diag}\left(\overline{\boldsymbol{\rho}}^{t}\right)\right)^{-1} \\
& \mathbf{B}^{t}=\mathbf{Z}_{I} \operatorname{diag}\left(\bar{\gamma}^{t}\right) \\
& \quad \times\left(\operatorname{diag}\left(\overline{\boldsymbol{\rho}}^{t}\right)\right)^{-1}+\mathbf{Z}_{R} \operatorname{diag}\left(\overline{\boldsymbol{\mu}}^{t}\right)\left(\operatorname{diag}\left(\overline{\boldsymbol{\rho}}^{t}\right)\right)^{-1}
\end{aligned}
$$

and $\mathbf{a}=\overline{\boldsymbol{\rho}}^{t}$, where $\mathbf{Z}_{R}:=\Re\left\{\mathbf{Y}^{-1}\right\}$ and $\mathbf{Z}_{I}:=\Im\left\{\mathbf{Y}^{-1}\right\}$. See [16] for more details. Equation (8) will be utilized next to develop a computationally affordable OPF strategy.

Remark 1 . The approximation (8) can be suitably modified to account for any value of the voltage angles $\bar{\theta}_{n}^{t}, n=$ $0,1, \ldots, N$. Particularly, a change of coordinates can be adopted to improve the approximation accuracy.

\section{RES AND BATTERY DISPATCH}

A multi-period OPF problem optimizing the operation of a distribution system over the interval $\mathcal{T}_{t}$ is formulated first, and subsequently utilized as a building block for a receding horizon control strategy. Particularly, at time instant $t$, the distribution system operator is assumed to have accurate knowledge of available powers $\mathbf{p}_{\mathrm{av}}^{t}$ and loads $\mathbf{p}_{\ell}^{t}, \mathbf{q}_{\ell}^{t}$; on the other hand, only a forecast is available for $\left\{\mathbf{p}_{\mathrm{av}}^{\tau}, \mathbf{p}_{\ell}^{\tau}, \mathbf{q}_{\ell}^{\tau}\right\}_{\tau=t+1}^{t+T}$. Consider then the following optimization problem to compute the optimal setpoints for RES and energy storage inverters:

$$
\begin{aligned}
& \text { (P0) } \min _{\substack{\boldsymbol{\rho}^{t},\left\{\boldsymbol{\alpha}^{\tau}, \mathbf{b}^{\tau}\right\} \\
\left\{\mathbf{p}_{B}^{\tau}\right\}}} C^{t}\left(\boldsymbol{\rho}^{t}, \boldsymbol{\alpha}^{t}, \mathbf{p}_{B}^{t}\right)+\sum_{\tau \in \mathcal{T}_{t}^{\prime}} C^{\tau}\left(\boldsymbol{\alpha}^{\tau}, \mathbf{p}_{B}^{\tau}, \mathbf{b}^{\tau}\right)(10 \mathrm{a}) \\
& \text { subject to } \\
& \boldsymbol{\rho}^{t}=\mathbf{g}_{\rho}\left(\boldsymbol{\alpha}^{t}, \mathbf{p}_{\mathrm{av}}^{t}, \mathbf{p}_{B}^{t}, \mathbf{p}_{\ell}^{t}, \mathbf{q}_{\ell}^{t}\right) \\
& V_{\text {min }} \leq \rho_{n}^{t} \leq V_{\text {max }} \quad \forall n \in \mathcal{N} \\
& \operatorname{Pr}\left\{g_{\rho, n}\left(\boldsymbol{\alpha}^{\tau}, \mathbf{p}_{\mathrm{av}}^{\tau}, \mathbf{p}_{B}^{\tau}, \mathbf{p}_{\ell}^{\tau}, \mathbf{q}_{\ell}^{\tau}\right) \leq V_{\max }\right\} \geq 1-\epsilon \\
& \forall n \in \mathcal{N}, \tau \in \mathcal{T}_{t}^{\prime}(10 \mathrm{~d}) \\
& \operatorname{Pr}\left\{V_{\min } \leq g_{\rho, n}\left(\boldsymbol{\alpha}^{\tau}, \mathbf{p}_{\mathrm{av}}^{\tau}, \mathbf{p}_{B}^{\tau}, \mathbf{p}_{\ell}^{\tau}, \mathbf{q}_{\ell}^{\tau}\right)\right\} \geq 1-\epsilon \\
& \forall n \in \mathcal{N}, \tau \in \mathcal{T}_{t}^{\prime}(10 \mathrm{e}) \\
& 0 \leq \alpha_{n}^{\tau} \leq 1 \\
& \forall n \in \mathcal{N}, \tau \in \mathcal{T}_{t} \\
& B_{n}^{\tau+1}=B_{n}^{\tau}+P_{B, n}^{\tau} \Delta^{t} \\
& \forall n \in \mathcal{N}, \tau \in \mathcal{T}_{t}^{\prime \prime} \\
& P_{B, n}^{\min } \leq P_{B, n}^{\tau} \leq P_{B, n}^{\max } \\
& \forall n \in \mathcal{N}, \tau \in \mathcal{T}_{t}^{\prime \prime}(10 \mathrm{~h}) \\
& B_{n}^{\text {min }} \leq B_{n}^{\tau} \leq B_{n}^{\max } \\
& \forall n \in \mathcal{N}, \tau \in \mathcal{T}_{t}^{\prime}
\end{aligned}
$$

where $g_{\rho, n}(\cdot)$ denotes the $n$-th element of the vector-valued function $\mathrm{g}_{\rho}(\cdot), \mathcal{T}_{t}^{\prime}:=\{t+1, \ldots, t+T\}$, and $\mathcal{T}_{t}^{\prime \prime}:=$ $\{t, \ldots, t+T-1\}$. Constraints (10b)-(10c) pertain to the current instant $t$, with (10b) representing a surrogate for the balance equation; and (10d)-(10i) optimize the RES and battery utilization over the whole horizon. Function $C^{t}\left(\boldsymbol{\rho}^{t}, \boldsymbol{\alpha}^{t}, \mathbf{p}_{B}^{t}\right)$ is convex and captures e.g., (cost of) power losses and real power curtailed from RESs [2], [3]; similarly, $\left\{C^{\tau}\left(\boldsymbol{\rho}^{\tau}, \boldsymbol{\alpha}^{\tau}, \mathbf{p}_{B}^{\tau}, \mathbf{b}^{\tau}\right)\right\}_{\tau \in \mathcal{T}_{t}^{\prime}}$ are convex functions that model e.g., (cost of) power losses, real power curtailed from RESs, cycling of batteries, and other economic performance indicators. Of particular relevance is the minimization of the real power curtailed, which promotes utilization of RESbased generation, while congruently respecting voltage limits. Finally, notice that under current modeling assumptions, approximations for the power losses that are convex and quadratic in the injected powers can be obtained.

Given the predicted values of both available powers $\left\{\overline{\mathbf{p}}_{\mathrm{av}}^{\tau}\right\}_{\tau \in \mathcal{T}_{t}^{\prime}}$ and loads $\left\{\overline{\mathbf{p}}_{\ell}^{\tau}, \overline{\mathbf{q}}_{\ell}^{\tau}\right\}_{\tau \in \mathcal{T}_{t}^{\prime}}$, along with the associated forecasting errors, the chance constraints (10d)-(10e) 
ensure that RES and battery setpoints can be scheduled in future time instants in a way that voltage limits are satisfied with prescribed probability. Constraints (10d)-(10e) are, however, problematic. Monte Carlo-based methods may lead to computationally heavy solution approaches. Furthermore, even if the function $g_{\rho, n}(\cdot)$ is affine, it turns out that the feasible set of (10d)-(10e) may be nonconvex. For example, (10d)-(10e) are convex and efficiently manageable only when $\boldsymbol{\delta}^{\tau}:=\left[\left(\mathbf{p}_{\mathrm{av}}^{\tau}\right)^{\top},\left(\mathbf{p}_{\ell}^{\tau}\right)^{\top},\left(\mathbf{q}_{\ell}^{\tau}\right)^{\top}\right]^{\top}$ is the image, under affine transformation, of a random vector with rotationally invariant distribution - with the multivariate Gaussian distribution as a prime example (see e.g., [15]).

To account for a variety of possible distributions of the forecasting errors $\boldsymbol{\delta}^{\tau}$ and yet derive a computationally efficient method for the stochastic multi-period OPF, a convex approximation of the chance constraints is pursued next.

\section{A. Leveraging convex approximation of chance constraints}

Consider the generic chance constraint $\operatorname{Pr}\{g(\mathbf{x}, \boldsymbol{\delta})>0\} \leq$ $\epsilon$, where function $g(\mathbf{x}, \boldsymbol{\delta})$ is convex in the optimization variables $\mathbf{x}$ for given values of the random vector $\boldsymbol{\delta}$, and $\epsilon$ is the maximum constraint violation rate. To develop a conservative convex approximation for this chance constraint, consider a function $\psi: \mathbb{R} \rightarrow \mathbb{R}$ that is nonnegative valued, nondecreasing, and convex. Further, assume that $\psi(\cdot)$ - henceforth referred to as the (one-dimensional) generating function satisfies the conditions $\psi(x)>\psi(0), \forall x>0$ and $\psi(0)=1$. Given a positive scalar $z>0$ and a random variable $\delta$, it holds that: $\mathbb{E}_{\delta}\left\{\phi\left(z^{-1} \delta\right)\right\} \geq \mathbb{E}_{\delta}\left\{\mathbb{I}_{[0,+\infty)}\left(z^{-1} \delta\right)\right\}=$ $\operatorname{Pr}\left\{z^{-1} \delta \geq 0\right\}=\operatorname{Pr}\{\delta \geq 0\}$, where $\mathbb{E}_{\delta}$ denotes expectation with respect to $\delta$. Thus, by taking $\delta=g(\mathbf{x}, \boldsymbol{\delta})$ one has that the following bound holds for all $z>0$ and $\mathbf{x}$ [15]:

$$
\operatorname{Pr}\{g(\mathbf{x}, \boldsymbol{\delta})>0\} \leq \mathbb{E}_{\boldsymbol{\delta}}\left\{\psi\left(z^{-1} g(\mathbf{x}, \boldsymbol{\delta})\right)\right\} .
$$

It follows that the constraint

$$
\inf _{z>0}\left\{z \mathbb{E}_{\boldsymbol{\delta}}\left\{\psi\left(z^{-1} g(\mathbf{x}, \boldsymbol{\delta})\right)-z \epsilon\right\}\right\} \leq 0
$$

represents a sufficient condition for $\operatorname{Pr}\{g(\mathbf{x}, \boldsymbol{\delta})>0\} \leq \epsilon$ and hence is also a conservative convex approximation of the chance constraint $\operatorname{Pr}\{g(\mathbf{x}, \boldsymbol{\delta}) \leq 0\} \leq 1-\epsilon$. Regarding the convexity of (12), notice that since $\psi(\cdot)$ is nondecreasing and convex and $g(\cdot, \boldsymbol{\delta})$ is convex, it follows that the mapping $(\mathbf{x}, z) \rightarrow z \psi\left(z^{-1} g(\mathbf{x}, \boldsymbol{\delta})\right)$ is convex. If $g$ is biaffine in $\mathbf{x}$ and $\boldsymbol{\delta}$ and $\psi$ is quadratic, then the constraint (12) is also convex.

Consider the piecewise linear function $\psi(x)=[1+x]_{+}$ to obtain the approximate constraint:

$$
\inf _{z \in \mathbb{R}} \mathbb{E}_{\boldsymbol{\delta}}\left\{[g(\mathbf{x}, \boldsymbol{\delta})+z]_{+}-z \alpha\right\} \leq 0
$$

where the infimum is taken over $z \in \mathbb{R}$ (instead of the non-negative orthant) without compromising the validity of the bound. It turns out that (13) represents the most accurate conservative approximation of the chance constraint $\operatorname{Pr}\{g(\mathbf{x}, \boldsymbol{\delta}) \leq 0\} \geq 1-\epsilon$ [15]. Further, (13) is closely related to the concept of conditional value at risk, which is a wellknown coherent risk measure in risk management and optimization under uncertainty [11], [14], [15]. Thus, replacing the generic convex function $g(\mathbf{x}, \boldsymbol{\delta})$ with $g_{\rho, n}\left(\boldsymbol{\alpha}^{\tau}, \mathbf{p}_{B}^{\tau}, \boldsymbol{\delta}^{\tau}\right)-$ $V_{\max }$ and $V_{\min }-g_{\rho, n}\left(\boldsymbol{\alpha}^{\tau}, \mathbf{p}_{B}^{\tau}, \boldsymbol{\delta}^{\tau}\right)$, respectively, it follows that Markov-based convex approximations of (10d)-(10e) amount to:

$$
\begin{aligned}
& \mathbb{E}_{\boldsymbol{\delta}^{\tau}}\left\{\left[g_{\rho, n}\left(\boldsymbol{\alpha}^{\tau}, \mathbf{p}_{B}^{\tau}, \boldsymbol{\delta}^{\tau}\right)-V_{\max }+z_{n}^{\tau}\right]_{+}\right\} \leq z_{n}^{\tau} \epsilon \\
& \mathbb{E}_{\boldsymbol{\delta}^{\tau}}\left\{\left[V_{\min }-g_{\rho, n}\left(\boldsymbol{\alpha}^{\tau}, \mathbf{p}_{B}^{\tau}, \boldsymbol{\delta}^{\tau}\right)+y_{n}^{\tau}\right]_{+}\right\} \leq y_{n}^{\tau} \epsilon
\end{aligned}
$$

where $\left\{z_{n}^{\tau} \in \mathbb{R}\right\}_{n, \tau}$ and $\left\{y_{n}^{\tau} \in \mathbb{R}\right\}_{n, \tau}$ will be auxiliary optimization variables. An advantage of (14) is that empirical estimates of the expected values can be obtained via sample averaging. Accordingly, given $S$ samples $\left\{\boldsymbol{\delta}^{\tau}[s]\right\}_{s=1}^{S}$, of the random vector $\boldsymbol{\delta}^{\tau}$, an approximation of (14) for arbitrary distributions can be accommodated in the OPF task as follows:

$$
\begin{gathered}
\text { (P1) } \min _{\begin{array}{c}
\boldsymbol{\rho}^{t},\left\{\boldsymbol{\alpha}^{\tau}, \mathbf{b}^{\tau}\right\} \\
\left\{\mathbf{p}_{B}^{\tau}\right\},\left\{z_{n}^{\tau}, y_{n}^{\tau}\right\}
\end{array}} C^{t}\left(\boldsymbol{\rho}^{t}, \boldsymbol{\alpha}^{t}, \mathbf{p}_{B}^{t}\right)+\sum_{\tau=t+1}^{t+T} C^{\tau}\left(\boldsymbol{\rho}^{\tau}, \boldsymbol{\alpha}^{\tau}, \mathbf{p}_{B}^{\tau}, \mathbf{b}^{\tau}\right) \\
\text { subject to (10b), (10c), (10f) - (10i), and } \\
\quad \forall n \in \mathcal{N}, \tau \in \mathcal{T}_{t}^{\prime} \\
\frac{1}{S} \sum_{s=1}^{S}\left[g_{\rho, n}\left(\boldsymbol{\alpha}^{\tau}, \mathbf{p}_{B}^{\tau}, \boldsymbol{\delta}^{\tau}[s]\right)-V_{\max }+z_{n}^{\tau}\right]_{+} \leq z_{n}^{\tau} \epsilon \\
\frac{1}{S} \sum_{s=1}^{S}\left[V_{\min }-g_{\rho, n}\left(\boldsymbol{\alpha}^{\tau}, \mathbf{p}_{B}^{\tau}, \boldsymbol{\delta}^{\tau}[s]\right)+y_{n}^{\tau}\right]_{+} \leq y_{n}^{\tau} \epsilon \\
\forall n \in \mathcal{N}, \tau \in \mathcal{T}_{t}^{\prime} .
\end{gathered}
$$

For a sufficiently high number of samples $S$, almost sure convergence of the sample averages in (15a)-(15b) to $\mathbb{E}_{\boldsymbol{\delta}^{\tau}}\left\{\left[g_{\rho, n}\left(\boldsymbol{\alpha}^{\tau}, \mathbf{p}_{B}^{\tau}, \boldsymbol{\delta}^{\tau}\right)-V_{\max }+z_{n}^{\tau}\right]_{+}\right\}$and $\mathbb{E}_{\boldsymbol{\delta}^{\tau}}\left\{\left[V_{\max }-\right.\right.$ $\left.\left.g_{\rho, n}\left(\boldsymbol{\alpha}^{\tau}, \mathbf{p}_{B}^{\tau}, \boldsymbol{\delta}^{\tau}\right)+y_{n}^{\tau}\right]_{+}\right\}$, respectively, is guaranteed by the (strong) law of large numbers. Regarding the approximate problem (15), the following points should be stressed: $i$ ) (P1) is a convex program; ii) the number of optimization variables does not increase with the increasing of the number of samples $S$; and, iii) any distribution of the random vectors $\mathbf{p}_{\mathrm{av}}^{\tau}, \mathbf{p}_{\ell}^{\tau}$, and $\mathbf{q}_{\ell}^{\tau}$ can be accommodated in (P1). Arbitrary distributions can be accommodated so long as one has a mechanism from which to draw samples of $\boldsymbol{\delta}_{\mathrm{av}}^{\tau}$ and $\boldsymbol{\delta}_{\ell}^{\tau}$.

Remark 2. For simplicity, no charging/discharging losses were considered in the dynamical equation of the energy storage (3) [13]. However, charging and discharging efficiencies can be readily incorporated in (3) and $(10 \mathrm{~g})$, at the cost of increasing the complexity of problems $(\mathrm{P} 0)-(\mathrm{P} 1)$. Letting $P_{B_{c}, n}^{t} \geq 0$ and $P_{B_{d}, n}^{t} \geq 0$ denote the power supplied to and withdrawn from the battery $n$, the equation (3) can be modified as:

$$
B_{n}^{t+1}=B_{n}^{t}+\eta_{c} P_{B_{c}, n}^{t} \Delta^{t}-\frac{1}{\eta_{d}} P_{B_{d}, n}^{t} \Delta^{t} .
$$

where $\eta_{c} \in(0,1]$ and $\eta_{d} \in(0,1]$ are the charging and discharging efficiencies, respectively. However, it is necessary to add the constraint $P_{B_{c}, n}^{t} P_{B_{d}, n}^{t}=0$ to avoid a solution where $P_{B_{c}, n}^{t}$ and $P_{B_{c}, n}^{t}$ are simultaneously nonzero. With this additional constraint problem (P1) becomes nonconvex. Successive convex approximation techniques can be utilized to identify a (possibly locally optimal) solution of (P1). 


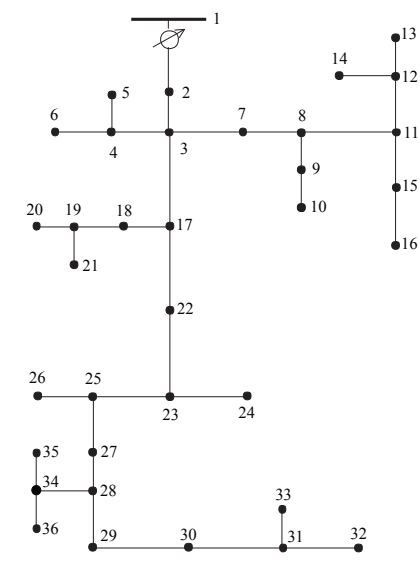

Fig. 1: Modified IEEE 37-node feeder.

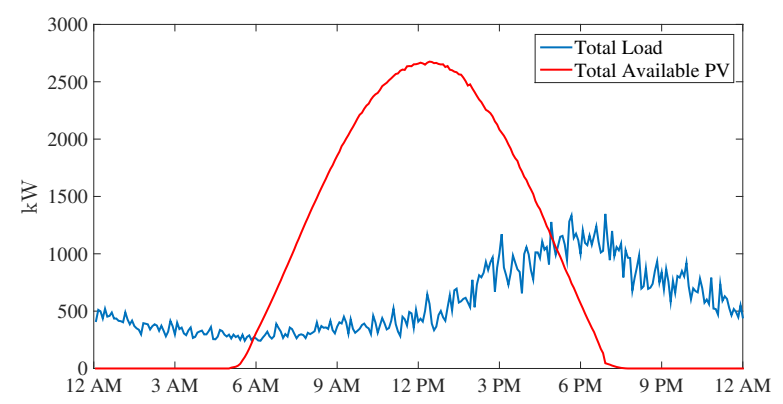

Fig. 2: Total feeder loading and available PV generation.

\section{B. Model predictive control implementation}

When determining the control decisions for devices with intertemporal constraints (e.g., energy storage units), it is advantageous to not only take into account the current time step, but also potential future system states. MPC is an adaptive control technique that enacts optimal control decisions in the current time step while taking into account the system behavior over a chosen time horizon [1], [5], [7]. In the present context, problem (P1) constitutes a building block for a MPC-based strategy. Particularly, the MPC strategy involves the following steps:

[S1] At time instant $t$, measure $\mathbf{p}_{\mathrm{av}}^{t}, \mathbf{p}_{\ell}^{t}$, and $\mathbf{q}_{\ell}^{t}$, and acquire the forecasting of available RES power and loads over $\mathcal{T}_{t}^{\prime}$.

[S2] Solve (P1) over the horizon $\mathcal{T}$.

[S3] Send setpoints $\left\{\left(1-\alpha_{n}^{t}\right) P_{\mathrm{av}, n}^{t}\right\}$ to the RES inverters, and $\left\{P_{B, n}^{t}\right\}$ to the battery inverters.

[S4] $\mathcal{T}_{t} \rightarrow \mathcal{T}_{t+1}$, and go to step [S1].

\section{NUMERICAL TESTS}

The proposed optimization scheme is tested using a modified version of the IEEE 37-node test feeder. The modified network is obtained by considering a single-phase equivalent, and by replacing the loads specified in the original dataset with real load data measured from a neighborhood called Anatolia in CA, during the month of August 2012 [19]. The total loading of the feeder can be seen in Figure 2, where data granularity is of 5 minutes. Line impedances, shunt admittances, as well as active and reactive loads are adopted from the respective dataset. It is assumed that twenty-one photovoltaic (PV) systems are located at nodes $4,7,9,10$, $11,13,16,17,20,22,23,26,28,29,30,31,32,33,34$, 35 , and 36 , and their generation profile is simulated based on the solar irradiance data available in [19]. The aggregate available power $\sum_{n} P_{\mathrm{av}, n}^{\tau}$ during the course of the day is plotted in Figure 2. The voltage limits $V_{\max }$ and $V_{\min }$ are set to $1.05 \mathrm{pu}$ and $0.95 \mathrm{pu}$, respectively. Without energy storage systems, and with RES-inverters injecting all the available power into the feeder, this configuration would lead to overvoltage conditions during peak irradiation hours.

Suppose that sixteen energy storage systems are co-located with PV systems at nodes $7,9,10,11,13,16,20,22,26$, $28,29,30,32,33,35$, and 36 , and their capacities $B_{n}^{\max }$ are 100, 200, 200,400,440,240,400, 240,300, 100, 500, $200,500,200,240,400,220,500,300,300$, and $300 \mathrm{kWh}$, respectively. In addition, $B_{n}^{\min }$ is set to zero for all batteries. Here, the cost function for the current time step is set to

$$
\begin{array}{r}
C^{t}\left(\boldsymbol{\rho}^{t}, \boldsymbol{\alpha}^{t}, \mathbf{p}_{B}^{t}\right)=\sum_{i \in \mathcal{N}} c_{i}^{t}\left[P_{\ell, i}^{t}+P_{B, i}^{t}-\left(1-\alpha_{i}^{t}\right) P_{\mathrm{av}, i}^{t}\right]_{+} \\
+\sum_{i \in \mathcal{N}} d_{i}^{t}\left[\left(1-\alpha_{i}^{t}\right) P_{\mathrm{av}, i}^{t}-P_{\ell, i}^{t}-P_{B, i}^{t}\right]_{+}
\end{array}
$$

where $c_{i}^{t}=10$ and $d_{i}^{t}=3$. Cost $C^{t}\left(\boldsymbol{\rho}^{t}, \boldsymbol{\alpha}^{t}, \mathbf{p}_{B}^{t}\right)$ captures the price associated with the power consumed by the customers, as well as the feed-in tariff cost to the utility. On the other hand, the cost $C^{\tau}\left(\boldsymbol{\rho}^{t}, \boldsymbol{\alpha}^{t}, \mathbf{p}_{B}^{t}, \mathbf{p}^{\tau}\right)$ for $\tau \in \mathcal{T}_{t}^{\prime}$ is set to $C^{\tau}\left(\boldsymbol{\alpha}^{t}, \mathbf{p}_{B}^{t}, \mathbf{p}^{\tau}\right)=\sum_{i \in \mathcal{N}} e_{i}^{\tau} \alpha_{i}^{\tau}$, with $e_{i}^{\tau}=3$, penalizing curtailment of real power from the PV systems. Unless otherwise specified, the parameter $\epsilon$ is fixed to 0.001 in the probabilistic constraints. The batteries were set to have a maximum five-minute charge rate of $10 \%$ of their respective capacities, $B_{n}^{\max }$. Forecasting errors for load and available real power are supposed to follow a truncated Gaussian distribution; the truncation is at $3 \sigma$, with $\sigma$ denoting the standard deviation. In particular, the standard deviation of the forecasting errors is assumed of $3 \%$ of the actual value for the first hour ahead and $7 \%$ for two to four hours ahead. Regarding the MPC strategy, two cases are considered: (C1) $T=2$ hours, with a granularity of 5 minutes during the first hour and 10 minutes during the second hour; and, (C2) $T=4$ hours, with a granularity of 15 minutes during the second hour and 30 minutes during the fourth hour. An MPC strategy implementing (P1) is considered. Finally, the solver SDPT3 is utilized to solve the optimization problems in MATLAB.

In Figure 3, the voltage profile without control and with the four-hour MPC strategy is shown. During peak irradiance hours, the system exhibits overvoltages if no PV power is curtailed and energy storage systems are not utilized. With the proposed method, the voltage profile flattens, becomes closer to nominal, and does not exceed voltage limits.

Figure 4 illustrates the state of charge of each of the energy storage systems throughout the twenty-four hour simulation period. As the value of the available PV power becomes higher than the total load, the batteries begin to charge. This avoids curtailing power as well as selling power back to the utility. Focusing on (C1), the achieved costs when $\epsilon$ is set to 0.001 and 0.1 are compared in Figure 5. As expected, 

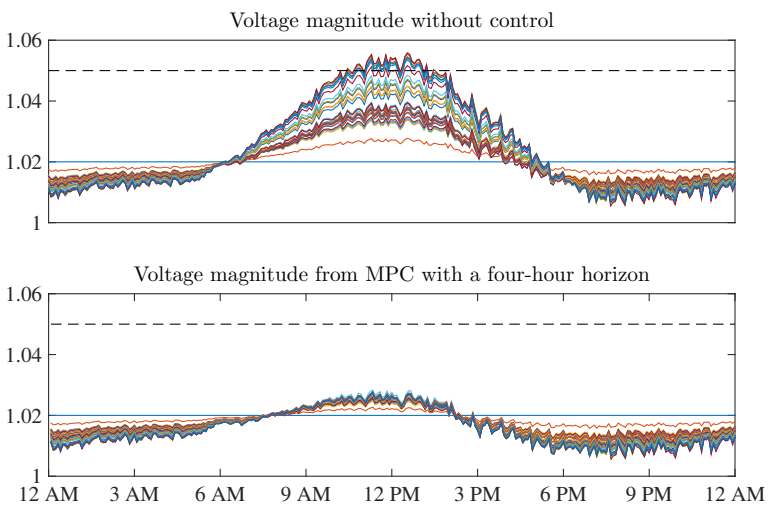

Fig. 3: Voltage profile achieved with the proposed strategy.

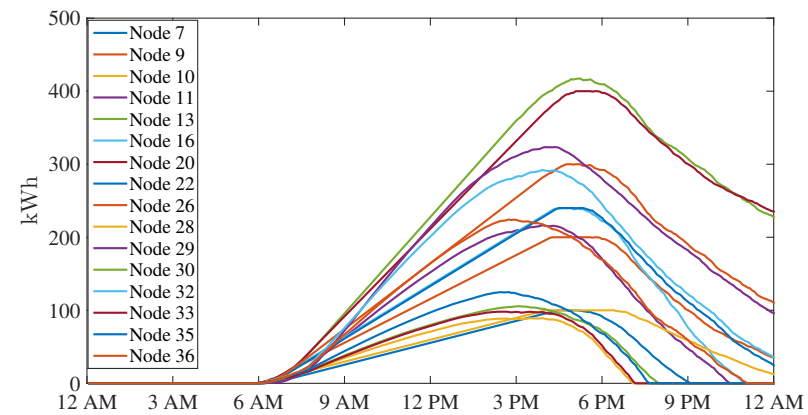

Fig. 4: Utilization profile of the energy storage systems for a four-hour horizon.

the cost becomes higher for a higher constraint fulfillment probability. A tradeoff between constraint violation and cost can be changed by modifying $\epsilon$.

\section{CONCLUDing REMARKS}

The paper developed an AC OPF approach to optimize the operation of distribution systems under forecasting errors. The proposed method utilizes a chance-constrained multiperiod AC OPF formulation, where probabilistic constraints are utilized to enforce voltage regulation with a prescribed probability. To enable a computationally affordable solution approach, an approximate reformulation of the considered $\mathrm{AC}$ OPF task is obtained by resorting to linear approximation of the AC power flow equations, and, conservative approximations for the chance constraints that hold for arbitrary distributions of the forecasting errors. The resultant problem is convex and efficiently solvable. An adaptive optimization strategy is then obtained via receding horizon control.

\section{REFERENCES}

[1] A. Parisio and L. Glielmo, "Stochastic model predictive control for economic/environmental operation management of microgrids," in IEEE European Control Conference, Zürich, Switzerland, 2013.

[2] E. Dall'Anese, S. Dhople, and G. Giannakis, "Optimal dispatch of photovoltaic inverters in residential distribution systems," IEEE Trans. Sust. Energy, vol. 5, no. 2, pp. 487-497, Apr. 2014.

[3] A. Samadi, R. Eriksson, L. Soder, B. G. Rawn, and J. C. Boemer, "Coordinated active power-dependent voltage regulation in distribution grids with PV systems," IEEE Trans. on Power Del., vol. 29, no. 3, pp. 1454-1464, June 2014.

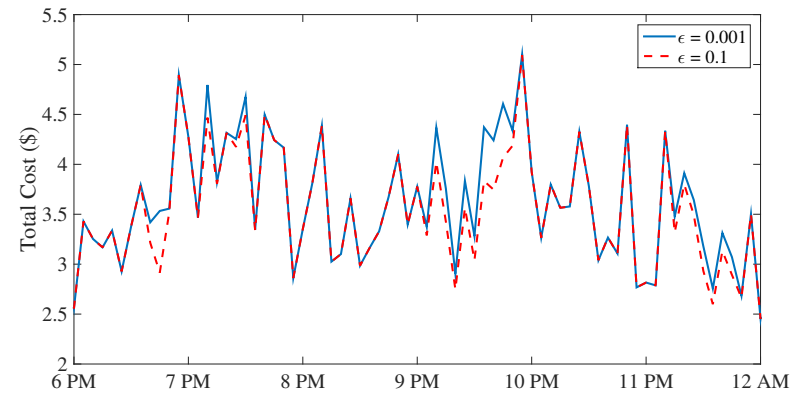

Fig. 5: Cost with varying constraint fulfillment parameter $\epsilon$.

[4] D. Bienstock, M. Chertkov, and S. Harnett, "Chance-constrained optimal power flow: Risk-aware network control under uncertainty," SIAM Review, vol. 56, no. 3, pp. 461-495, 2014.

[5] P. Fortenbacher, A. Ulbig, S. Koch, and G. Andersson, "Gridconstrained optimal predictive power dispatch in large multi-level power systems with renewable energy sources, and storage devices," in IEEE PES ISGT Europe, Istanbul, Turkey, Oct. 2014.

[6] A. Nagarajan and R. Ayyanar, "Design and strategy for the deployment of energy storage systems in a distribution feeder with penetration of renewable resources," IEEE Transactions on Sustainable Energy, vol. 6, no. 3, pp. 1085-1092, 2015.

[7] F. Adamek, M. Arnold, and G. Andersson, "On decisive storage parameters for minimizing energy supply costs in multicarrier energy systems," IEEE Trans. on Sus. Energy, vol. 5, no. 1, pp. 102-109, Jan 2014.

[8] K. Baker, J. Guo, G. Hug, and X. Li, "Distributed MPC for efficient coordination of storage and renewable energy sources across control areas," IEEE Transactions on Smart Grid, Special issue on Distributed Energy Management Systems, vol. 7, no. 2, pp. 992-1001, March 2016.

[9] R. A. Jabr, S. Karaki, and J. A. Korbane, "Robust multi-period OPF with storage and renewables," IEEE Trans. Power Syst., vol. 30, no. 5, pp. 2790-2799, Sept 2015

[10] J. Warrington, P. Goulart, S. Mariethoz, and M. Morari, "Policy-based reserves for power systems," IEEE Trans. on Power Systems, vol. 28 , no. 4, pp. 4427-4437, Nov 2013.

[11] T. Summers, J. Warrington, M. Morari, and J. Lygeros, "Stochastic optimal power flow based on conditional value at risk and distributional robustness," International Journal of Electrical Power \& Energy Systems, vol. 72, pp. 116-125, Nov. 2015.

[12] L. Roald, S. Misra, M. Chertkov, and G. Andersson, "Optimal power flow with weighted chance constraints and general policies for generation control," 2015, [Online] Available at: http://arxiv.org/pdf/1504.00057.

[13] D. Gayme and U. Topcu, "Optimal power flow with large-scale storage integration," IEEE Trans. Power Syst., vol. 28, pp. 709-717, 2012.

[14] E. Dall'Anese, S. Dhople, B. Johnson, and G. Giannakis, "Optimal dispatch of residential photovoltaic inverters under forecasting uncertainties," IEEE J. of Photovoltaics, vol. 5, no. 1, pp. 350-359, Jan 2015.

[15] A. Nemirovski and A. Shapiro, "Convex approximations of chance constrained programs," SIAM J. on Optimization, vol. 17, no. 4, pp. 969-996, 2007.

[16] S. Dhople, S. Guggilam, and Y. Chen, "Linear approximations to $\mathrm{AC}$ power flow in rectangular coordinates," Allerton Conference on Communication, Control, and Computing, in Press, 2015.

[17] W. H. Kersting, Distribution System Modeling and Analysis. 2nd ed., Boca Raton, FL: CRC Press, 2007.

[18] J. Lavaei and S. Low, "Zero duality gap in optimal power flow problem," IEEE Trans. Power Syst., vol. 1, no. 1, pp. 92-107, Feb. 2012

[19] J. Bank and J. Hambrick, "Development of a high resolution, real time, distribution-level metering system and associated visualization modeling, and data analysis functions," National Renewable Energy Laboratory, Tech. Rep. NREL/TP-5500-56610, May 2013. 